\title{
KNDC1 knockdown protects human umbilical vein endothelial cells from senescence
}

\author{
CHUNYAN ZHANG ${ }^{1 *}$, YONG-ZHAN ZHEN ${ }^{2 *}$, YA-JUN LIN ${ }^{3}$, JIANG LIU $^{4}$, JIE WEI $^{3}$, RONG XU $^{3}$ and GANG HU ${ }^{3}$ \\ ${ }^{1}$ Radiologic Department, Beijing Shijitan Hospital Affiliated of Capital Medical University, Beijing 100038; \\ ${ }^{2}$ Department of Histology and Embryology, Basic Medical College of Hebei United University, Tangshan, Hebei 063000; \\ ${ }^{3}$ Key Laboratory of Geriatrics, Beijing Hospital and Beijing Institute of Geriatrics, Ministry of Health, Beijing 100730; \\ ${ }^{4}$ Department of Endocrinology, The Third Hospital of Nanchang City, Nanchang, Jiangxi 330009, P.R. China
}

Received August 29, 2013; Accepted April 7, 2014

DOI: $10.3892 / \mathrm{mmr} .2014 .2201$

\begin{abstract}
KNDC1 (kinase noncatalytic C-lobe domain containing 1), a brain-specific Ras guanine nucleotide exchange factor, controls the negative regulation of neuronal dendrite growth. However, the effect of KNDC1 on cellular senescence remains to be elucidated. The present study investigated the impact of KNDC1 knockdown on human endothelial cell senescence and the mechanisms underlying this effect. Human umbilical vein endothelial cells (HUVECs) cultured in vitro were used as a model of biological aging. Senescence-associated $\beta$-galactosidase staining was used to detect cellular senescence and flow cytometry was employed to determine cell cycle progression. Quantitative polymerase chain reaction (qPCR) and western blot analysis were utilized to investigate mRNA transcription and protein expression. In the HUVECs, a senescence-like phenotypes developed with increasing passage number in vitro, which were associated with a progressive increase in the transcription and expression of KNDC1. KNDC1 knockdown promoted cell proliferation and partially reversed cellular senescence and cell cycle arrest in the G0/G1 phase in aging HUVECs. Investigations into the mechanism underlying this effect demonstrated that KNDC1 knockdown promoted HUVEC proliferation via the extracellular signal-regulated kinase signaling pathway and delayed HUVEC senescence by inhibiting the p53-p21-p16 transduction cascade. In addition, the promotion of the capillary tube network formation and the increased expression of endothelial nitric oxide synthase revealed that the activity and function
\end{abstract}

Correspondence to: Professor Gang Hu, Key Laboratory of Geriatrics, Beijing Hospital and Beijing Institute of Geriatrics, Ministry of Health, Room 1010, 1 Dahua Road, Dongdan, Dongcheng, Beijing 100730, P.R. China

E-mail: linyajun2000@126.com

*Contributed equally

Key words: kinase noncatalytic C-lobe domain containing 1, knockdown, human umbilical vein endothelial cells, cellular senescence of endothelial cells were enhanced. In conclusion, KNDC1 knockdown delayed endothelial cell senescence and promoted HUVEC activity and function. These results demonstrated that KNDC1 may be a novel therapeutic target for the development of agents to extend human life.

\section{Introduction}

Cellular senescence is a process of cellular aging in which primary cells in culture lose their ability to divide (1). It has been demonstrated that senescence is associated with cellular dysfunction and occurs in vivo in cardiovascular diseases associated with age, such as atherosclerosis $(2,3)$. In endothelial cells, the senescence-induced loss of replicative capacity destroys the integrity of the endothelium and impairs successful angiogenesis $(4,5)$.

In several recent studies, protein-protein interactions have been demonstrated to be important in the molecular recognition and functional modulation of proteins in numerous signal transduction pathways $(6,7)$. The kinase non-catalytic C-lobe domain (KIND) is a putative protein-protein interaction module (8). Four KIND-containing proteins have been reported: Spir-2 (an actin-nucleation factor), PTPN13 (a protein tyrosine phosphatase), FRMPD2 (a scaffold protein) and the Ras guanine exchange factor (RasGEF), very-KIND [v-KIND, also termed kinase noncatalytic C-lobe domain containing 1, (KNDC1)] (9,10). v-KIND, a brain-specific Ras guanine nucleotide exchange factor, has two KIND isoforms, KIND1 and KIND2, whereas the other three proteins have only one. A previous study demonstrated that v-KIND interacts with the high-molecular weight microtubule associated protein 2 (MAP2), a dendritic protein that drives negative regulation of neuronal dendrite growth. v-KIND overexpression suppresses the growth and branching of the dendrites of hippocampal neurons and cerebellar granule cells, whereas knockdown of endogenous v-KIND expression promotes dendrite growth. These findings suggest that v-KIND regulates a signaling pathway that links Ras and MAP2 to control dendrite growth (11). However, its role in vascular cell biology has not been investigated to date.

In the present study, the expression of KNDC1 with increasing age, as well as the effects of its depletion by RNA 
interference on senescence were investigated in human umbilical vein endothelial cells (HUVECS).

\section{Materials and methods}

Chemicals and reagents. Trypsin was purchased from Invitrogen Life Technologies (Beijing, China). Antibodies against extracellular signal-regulated kinase (ERK) $1 / 2$, phospho-ERK1/2 (Thr202/Tyr204; rabbit polyclonal), p38, phospho-p38 (Thr180/Tyr182; mouse monoclonal), stress-activated protein kinase (SAPK)/c-Jun N-terminal kinase (JNK), phospho-SAPK/JNK (Thr183/Tyr185; rabbit monoclonal), endothelial nitric oxide synthase (eNOS; rabbit polyclonal), vascular cell adhesion molecule (VCAM-1; rabbit polyclonal), intercellular adhesion molecule (ICAM-1; rabbit polyclonal), p53, phospho-p53 (Ser46; rabbit polyclonal), p21 (mouse monoclonal) and p16 (rabbit polyclonal) were purchased from Cell Signaling Technology, Inc. (Cell Signaling Technology, Danvers, MA, USA). Antibodies against KNDC1 and $\beta$-actin were purchased from Santa Cruz Biotechnology, Inc. (Santa Cruz Biotechnology, Inc., Santa Cruz, CA, USA). Secondary antibodies against rabbit and mouse were purchased from Cell Signaling Technology, Inc. The pre-stained protein marker was purchased from New England Biolabs, Ltd. (Beijing, China). Luminol reagent and polyvinylidene fluoride (PVDF) membrane for western blotting were purchased from Millipore (Millipore, Billerica, MA, USA).

Cells and cell culture. HUVECs were isolated from the umbilical cords of newborns supplied by Tongren Hospital (Beijing, China) and grown in M199 cell medium (Hyclone, Logan, UT, USA) containing $100 \mathrm{mg} / \mathrm{ml}$ streptomycin, $100 \mathrm{IU} / \mathrm{ml}$ penicillin, $40 \mu \mathrm{g} / \mathrm{ml}$ endothelial cell growth supplement and $20 \%$ fetal bovine serum (Hyclone) at $37^{\circ} \mathrm{C}$ in a humidified atmosphere of $95 \%$ air and $5 \% \mathrm{CO}_{2}$. The cells were passaged at $80-90 \%$ confluence at a ratio of $1: 2$ and used for experiments at passages $(\mathrm{P})$ 3-5.

Transfections. The fourth-passage HUVECs were transfected at $70 \%$ confluence for $24 \mathrm{~h}$ with $20 \mathrm{nM}$ small interfering (si)RNAs targeting human KNDC1 [KNDC1siRNA1 was obtained from Santa Cruz Biotechnology, Inc. (SC-90387); KNDC1-siRNA2 was obtained from Invitrogen Life Technologies and the sense sequence was as follows: CAUCCAGGAGGAAUUUGCCUUUGAU]. A non-targeting control pool (NT-siRNA; Santa Cruz Biotechnology, Inc.) was also used. Transfections were performed using Hyperfect reagent (Qiagen, Shanghai, China) according to the manufacturer's instructions. After $4 \mathrm{~h}$, fresh medium was added and the cells were cultured for a further 72 -h period prior to analysis.

Senescence-associated $\beta$-galactosidase (SA- $\beta$-gal) staining. Endothelial cells were transfected with KNDC1-siRNA or NT-siRNA. Following incubation for $72 \mathrm{~h}$, the cells were washed twice with phosphate-buffered saline (PBS) and then fixed for 5 min with PBS containing 2\% formaldehyde and $0.2 \%$ glutaraldehyde. The cells were then incubated at $37^{\circ} \mathrm{C}$ for $10 \mathrm{~h}$ in a staining solution of $40 \mathrm{mM}$ citric acid, sodium phosphate, $\mathrm{pH} 6.0,1 \mathrm{mg} / \mathrm{ml}$ 5-bromo-4-chloro-3-isolyl$\beta$-D-galactoside (X-gal; Sigma, Shanghai, China), $5 \mathrm{mM}$ potassium ferrocyanide, $5 \mathrm{mM}$ potassium ferricyanide, $150 \mathrm{mM} \mathrm{NaCl}$ and $2 \mathrm{mM} \mathrm{MgCl}{ }_{2}$. SA- $\beta$-gal-positive cells were observed by microscopy (CKX31; Olympus, Beijing, China) and over 400 cells were counted in three independent fields as described previously (12).

RNA expression analysis. Cellular RNA was extracted with TRIzol reagent (Invitrogen Life Technologies) according to the manufacturer's instructions. RNA expression was measured by quantitative polymerase chain reaction (qPCR) with the appropriate primers using the one step SYBR PrimeScript RT-PCR kit (Takara Bio, Inc., Dalian, China). A $20 \mu$ l PCR reaction mixture was initially amplified and primer pairs for KNDC1 were obtained from Santa Cruz Biotechnology, Inc. Primer pairs for $\beta$-actin were synthesized by Shanghai Bioengineering Company (Shanghai, China). The PCR was run on an iCycler (Bio-Rad, Hercules, CA, USA). The thermal profile for SYBR qPCR was $42^{\circ} \mathrm{C}$ for $5 \mathrm{~min}, 95^{\circ} \mathrm{C}$ for $10 \mathrm{sec}$ followed by 40 amplification cycles of $95^{\circ} \mathrm{C}$ for $5 \mathrm{sec}$ and $60^{\circ} \mathrm{C}$ for $20 \mathrm{sec}$. Relative mRNA expression levels were calculated by the comparative cycle threshold (CT) method, using the CT values obtained for $\beta$-actin as internal references.

Western blot analysis. Endothelial cells $\left(7.5 \times 10^{5}\right)$ were washed with ice-cold PBS and scraped off the flask into $100 \mu \mathrm{l}$ lysis buffer containing $25 \mathrm{mM}$ Tris- $\mathrm{HCl}, \mathrm{pH}$ 6.8, $1 \%$ sodium dodecylsulfate, $1 \mathrm{mM}$ phenylmethylsulphonyl fluoride and protease inhibitor cocktail (Sigma). The resultant lysates were further disrupted by sonication for $10 \mathrm{sec}$ at an amplitude of $35 \%$ using a VCX 500 Ultrasonic Processor (Sonics \& Materials, Newtown, CT, USA) and then centrifuged at 12,000 x g for $20 \mathrm{~min}$ to remove particulate material. Proteins $(30 \mu \mathrm{g})$ were fractionated by SDS-PAGE and transferred to PVDF membranes. The membranes were incubated as previously described (12).

Cell cycle analysis. Cells $\left(4 \times 10^{5}\right)$ were plated in $25 \mathrm{~cm}^{2}$ flasks and cultured in M199 medium. Following incubation for $24 \mathrm{~h}$, the cells were transfected with KNDC1-siRNA or NT-siRNA. The cells were harvested at $72 \mathrm{~h}$ following transfection, washed twice in salt buffer (1\% bovine serum albumin and $0.5 \%$ sodium azide in PBS) and fixed in $70 \%$ ethanol at $4^{\circ} \mathrm{C}$ overnight. After washing twice in salt buffer, the cells were stained in $50 \mu \mathrm{g} / \mathrm{ml}$ propidium iodide (PI) solution (Sigma) containing $100 \mu \mathrm{g} / \mathrm{ml}$ RNase for $1 \mathrm{~h}$. Then, the cells were transferred to flow cytometry tubes with filters for cell cycle analysis.

Capillary tube network formation. Endothelial capillary tube network formation was assessed using Matrigel (BD Biosciences, Franklin Lakes, NJ, USA). Each well of the 96-well culture plates was coated with $100 \mu \mathrm{l}$ Matrigel and immediately incubated at $37^{\circ} \mathrm{C}$ for $1 \mathrm{~h}$ to allow gel formation. Upon gelation, transfected cells were seeded onto the Matrigel-coated 96-well plates $\left(3 \times 10^{4}\right.$ cells/well) in $100 \mu 1$ M199 medium. Following incubation for 24 h, tubule formation was analyzed under an Olympus inverted microscope (CKX31; Olympus, Beijing, China) at a magnification of x40. Images were captured under phase contrast using an Olympus digital camera and analyzed with the Image J software 

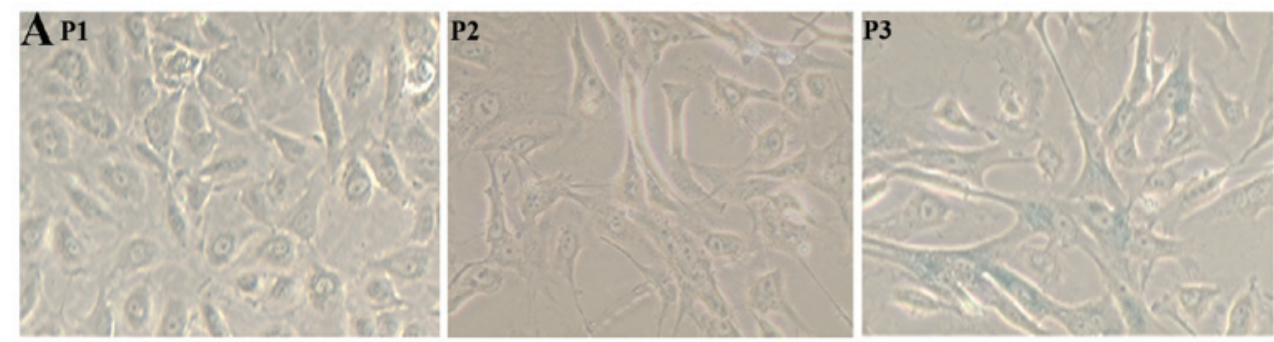
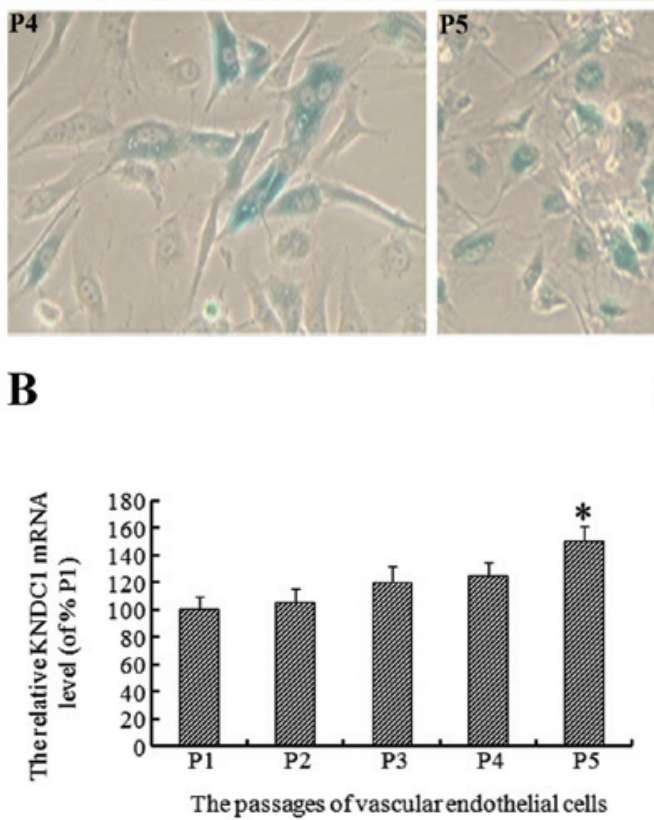
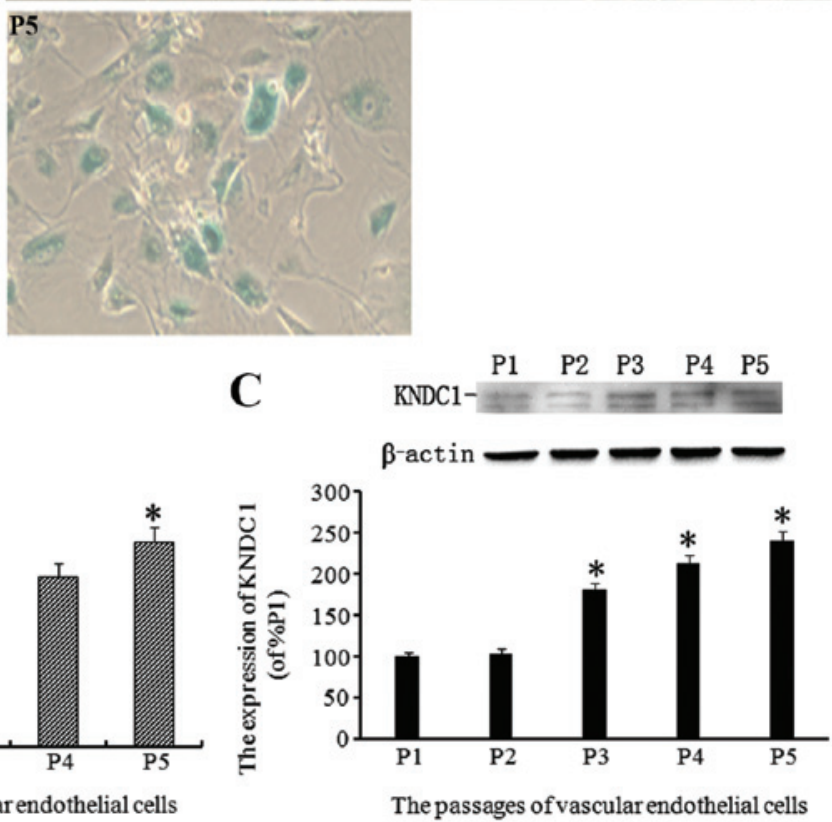

Figure 1. Development of a senescence-like phenotype and increased transcription and expression of KNDC1 in HUVECs with increasing passage number in vitro. (A) The percentage of senescent HUVECs at different passages was determined by senescence-associated $\beta$-galactosidase staining (magnification, x200). (B) KNDC1 transcription was analyzed by quantitative polymerase chain reaction. (C) KNDC1 expression was analyzed by western blotting ( $\mathrm{n}=3$ ). Values are presented as the mean \pm standard deviation. "P<0.05 vs $\mathrm{P} 1$. KNDC1, kinase noncatalytic C-lobe domain containing 1; HUVECs, human umbilical vein endothelial cells; P, passage.

(National Institutes of Health, Bethesda, MD, USA). Tubule length was determined by drawing a line along each tubule and measuring the line length in pixels. Branch points were counted manually.

Statistical analysis. Values are presented as the mean \pm standard deviation. Statistical analysis was performed using SPSS 11.0 (SPSS, Inc., Chicago, IL, USA). Results were evaluated by t-test or one-way analysis of variance followed by Bonferroni's post-hoc tests as appropriate. A value of $\mathrm{P}<0.05$ was considered to indicate a statistically significant difference.

\section{Results}

Senescence-like phenotype and increased transcription and expression of KNDC1 in HUVECs with increasing passage number. When HUVECs are cultured in vitro, their growth slows down and proliferation stops at the fifth passage. In the present study, it was observed that with increasing passage number, the number of aging HUVEC cells increased, as demonstrated by SA- $\beta$-gal staining. The percentages of aging cells at different passages were $\sim 2.0 \pm 2.0 \%$ (P1), $8.0 \pm 3.0 \%$ (P2), $15.0 \pm 3.0 \%(\mathrm{P} 3), 35.0 \pm 6.0 \%(\mathrm{P} 4)$ and $62.0 \pm 10.0 \%$ (P5; Fig. 1A). To investigate whether KNDC1 was associated with the senescence of normal cells, the expression levels of KNDC1 in HUVECs at different passages were examined by qPCR and western blot analysis. The levels of KNDC1 mRNA exhibited a statistically significant increase in HUVECs at P1-P5 (Fig. 1B). Similarly, the levels of KNDC1 protein also demonstrated a statistically significant increase in P1-P5 HUVECs (Fig. 1C).

Partial reversal of cellular senescence in aging HUVECs by KNDCl knockdown. Aging cells express SA- $\beta$-gal, are resistant to mitogen-induced proliferation and have a characteristically enlarged and flattened morphology. In the present study, HUVECs at P4 exhibited senescence phenotypes that distinguished them from early passage cells. To investigate the role of KNDC1 in cellular senescence, the levels of KNDC1 mRNA and protein in HUVECs at $\mathrm{P} 4$ were downregulated by gene silencing with KNDC1-siRNA1/2. Transfection with KNDC1-siRNA1/2 caused a 50\% decrease in KNDC1 levels (Fig. 2A and B). In subsequent studies, only KNDC1siRNA1 was used, as KNDC1-siRNA1 and -2 had the same effect. Following repression of KNDC1 levels in HUVECs at $\mathrm{P} 4$, the size of the cells was similar to the size of P1 cells and the activity of SA- $\beta$-gal was decreased compared with that in NT-siRNA-transfected cells (10.0 3 vs. $38.0 \pm 7 \%$; Fig. $2 \mathrm{C}$ and D). 

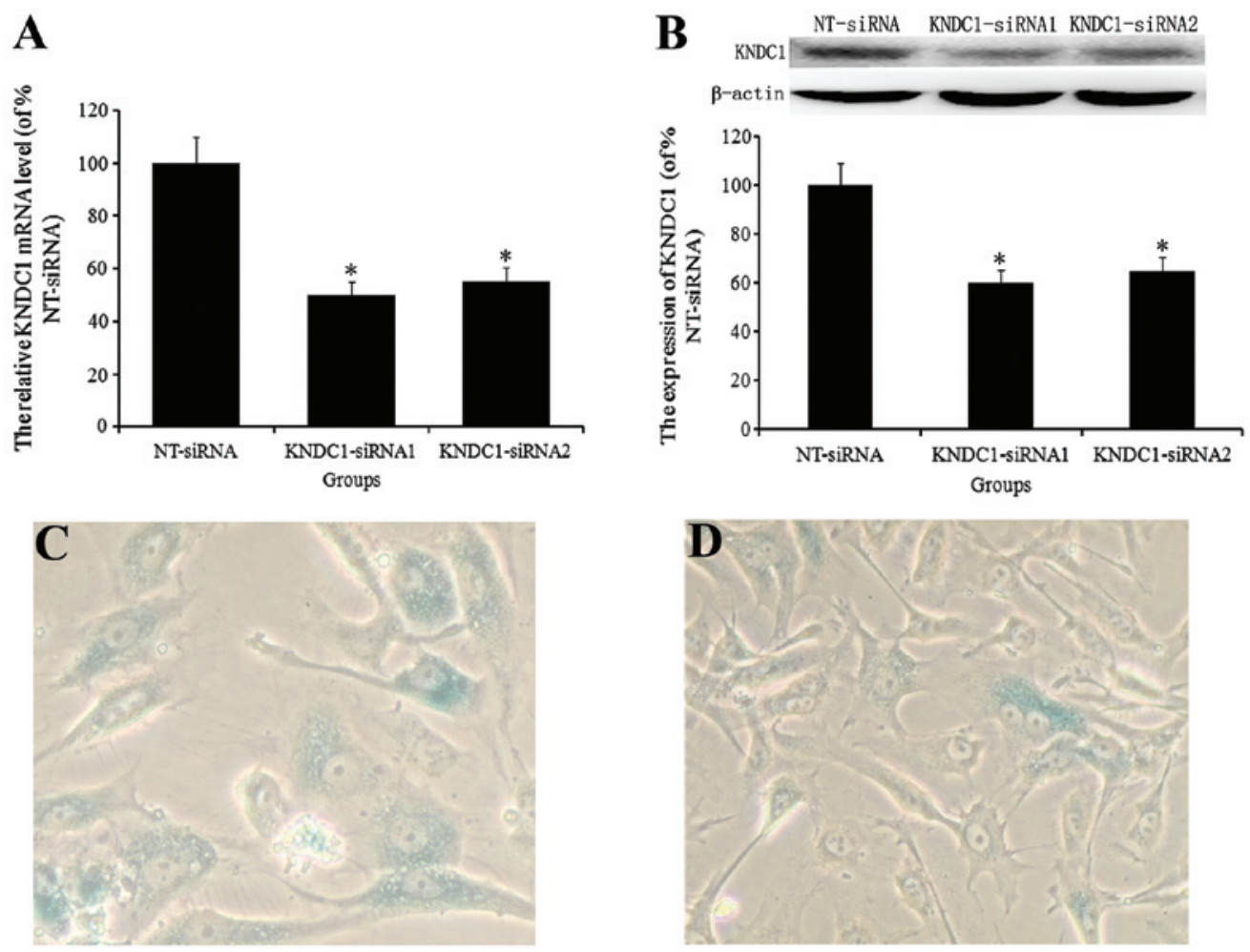

Figure 2. Knockdown of KNDC1 promotes cell proliferation and partially reverses cellular senescence in aging HUVECs. Following transfection with NT-siRNA or KNDC1-siRNA, the expression levels of KNDC1 mRNA and protein were determined by (A) quantitative polymerase chain reaction and (B) western blotting. The percentage of senescent HUVECs transfected with (C) NT-siRNA or (D) KNDC1-siRNA was determined by senescence-associated $\beta$-galactosidase staining $(\mathrm{n}=3$; magnification, $\mathrm{x} 200)$. Values are presented as the mean \pm standard deviation. ${ }^{*} \mathrm{P}<0.05$ vs NT-siRNA. KNDC1, kinase noncatalytic C-lobe domain containing 1; HUVECs, human umbilical vein endothelial cells; NT-siRNA, non-targeting control pool; siRNA, small interfering RNA.

A

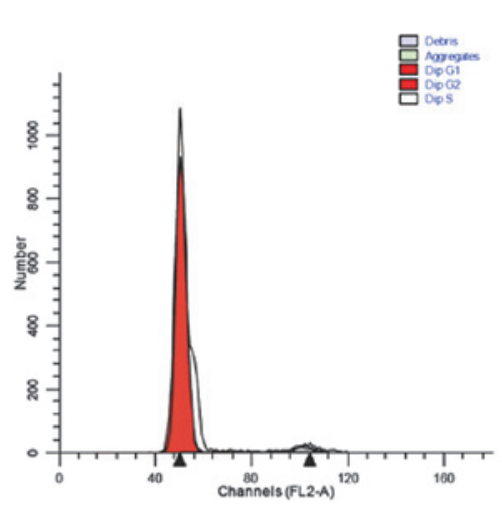

C



B

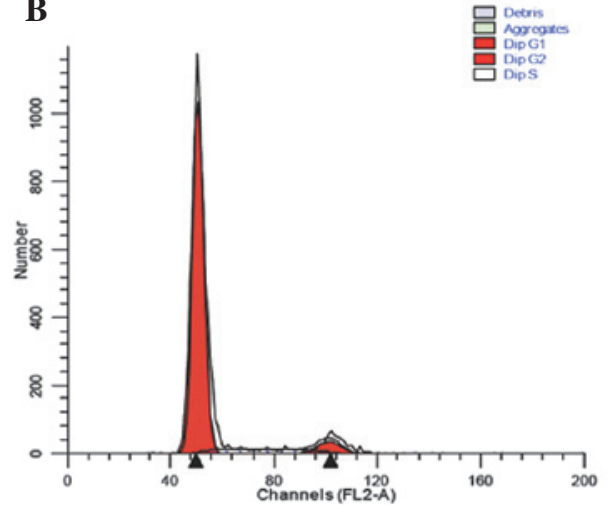

D

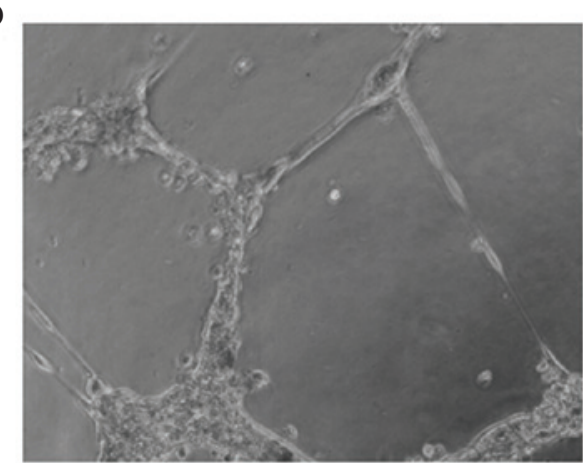

Figure 3. Changes in cell cycle progression and capillary tube network formation following knockdown of KNDC1. Following transfection with (A) KNDC1-siRNA or (B) NT-siRNA, the cell cycle was analyzed by flow cytometry with propidium iodide staining. HUVECs transfected with (C) KNDC1-siRNA or (D) NT-siRNA

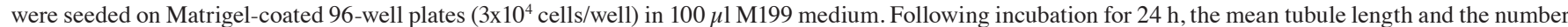
of branch points were calculated under an Olympus inverted microscope (magnification, x40). KNDC1, kinase noncatalytic C-lobe domain containing 1; HUVECs, human umbilical vein endothelial cells; NT-siRNA, non-targeting control pool; siRNA, small interfering RNA. 
Table I. Cell cycle distribution of P4 generation HUVECs following KDNC1-siRNA treatment for $72 \mathrm{~h}(\mathrm{n}=3)$.

\begin{tabular}{lccc}
\hline & \multicolumn{3}{c}{ Percentage (\%) } \\
\cline { 2 - 4 } Groups & $\mathrm{G} 1$ & $\mathrm{~S}$ & $\mathrm{G} 2 / \mathrm{M}$ \\
\hline NT-siRNA & $94.25 \pm 5.6$ & $4.75 \pm 1.0$ & $1.00 \pm 0.5$ \\
KNDC1-siRNA & $85.78 \pm 4.5^{*}$ & $8.29 \pm 1.0^{*}$ & $5.93 \pm 1.0^{*}$ \\
\hline
\end{tabular}

Data are presented as the mean \pm standard deviation. ${ }^{*} \mathrm{P}<0.05$ compared with NT-siRNA. KNDC1, kinase noncatalytic C-lobe domain containing 1; HUVECs, human umbilical vein endothelial cells; P4, passage 4; NT-siRNA, non-targeting control pool; siRNA, small interfering RNA.
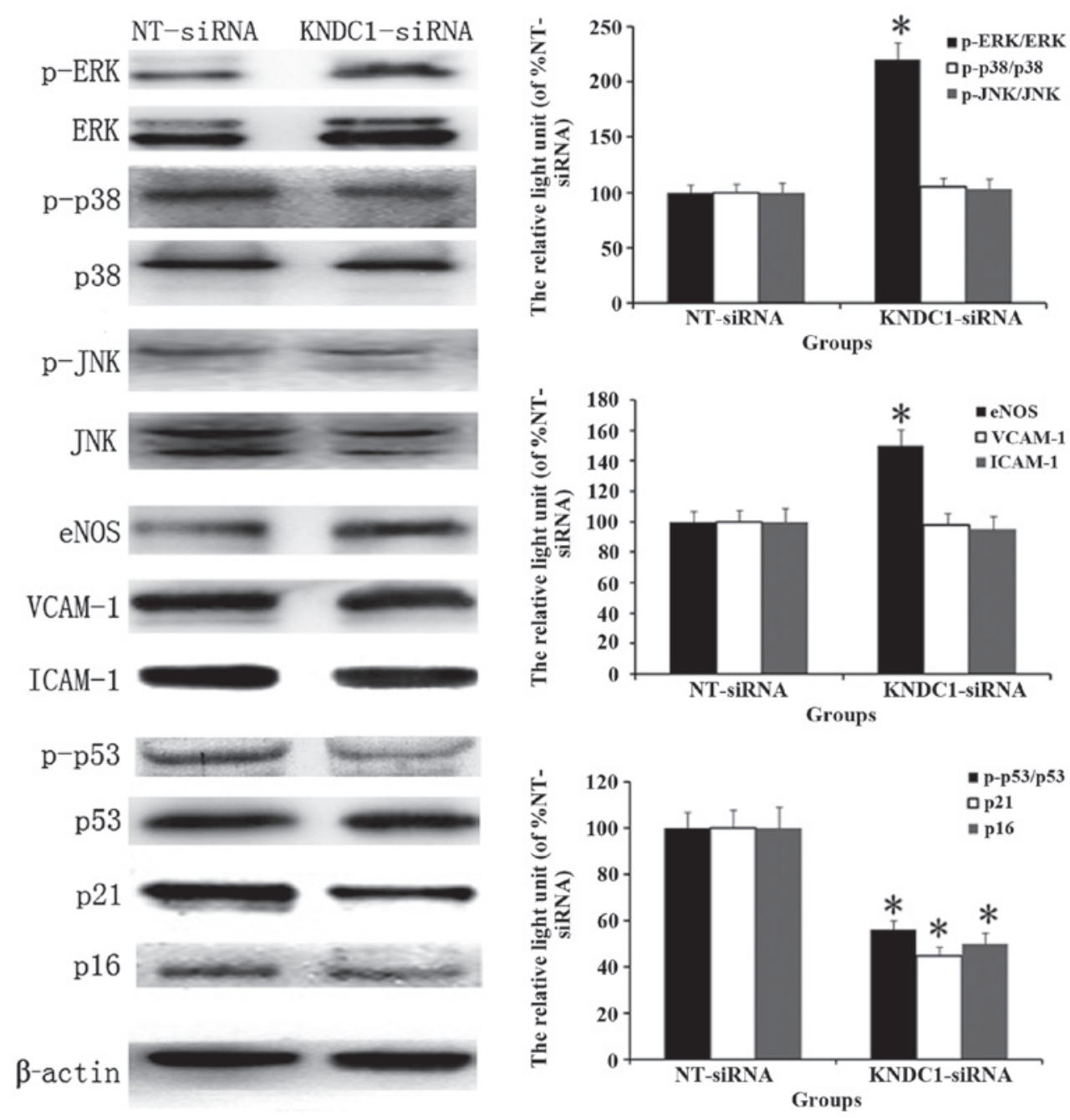

Figure 4. Effect of KNDC1 knockdown on signaling pathways. HUVECs were transfected with KNDC1-siRNA or NT-siRNA. Following incubation for $72 \mathrm{~h}$, the expression levels of eNOS, VCAM-1, ICAM-1, p21, p16 and p-ERK, p38, JNK and p53 were examined by western blot analysis (n=3). Values are presented as the mean \pm standard deviation. ${ }^{*} \mathrm{P}<0.05$ vs NT-siRNA. KNDC1, kinase noncatalytic C-lobe domain containing 1 ; HUVECs, human umbilical vein endothelial cells; NT-siRNA, non-targeting control pool; p, phosphorylated; siRNA, small interfering RNA; ERK, extracellular signal-regulated kinase; JNK, c-Jun N-terminal kinase; eNOS, endothelial nitric oxide synthase; VCAM-1, vascular cell adhesion molecule 1; ICAM-1, intercellular adhesion molecule 1.

Knockdown of KNDCl affects cell cycle progression. Replicatively senescent cells are known to undergo arrest in the G1/G0 phase of the cell cycle. To investigate whether cell proliferation induced by KNDC1-siRNA impacted the cell cycle, HUVECs at P4 were transfected with KNDC1-siRNA or NT-siRNA for $72 \mathrm{~h}$ and their DNA content was quantified by flow cytometry with PI staining. The majority of the cells transfected with NT-siRNA for $72 \mathrm{~h}$ had undergone G1 phase arrest, which is one of the typical phenotypes of cellular senescence. However, the number of cells in G1 phase was decreased in cells transfected with KNDC1-siRNA (Fig. 3A and B; Table I).

Knockdown of KNDC1 improves capillary tube network formation. Since senescence is associated with impaired angiogenic function, it was examined whether KNDC1 
knockdown improved the ability of HUVECs to form capillary tube networks in vitro. Compared with NT-siRNA-treated cells, KNDC1-silenced cells formed more developed tubule networks, in which the mean tubule length $(15 \pm 5.2 \mathrm{~mm}$ vs. $30 \mathrm{~mm} \pm 7.0)$ and the number of branches at each point (3 $\pm 1.0 /$ point vs. $2 \pm 1.0 /$ point) increased significantly (Fig. 3C and D).

Alterations in signaling pathways induced by knockdown of $K N D C 1$. In the present study, the effect of KNDC1 knockdown on the proliferation of endothelial cells [mitogen-activated protein kinase (MAPK) signaling pathway], the function of endothelial cells (eNOS, VCAM-1 and ICAM-1) and the senescence of endothelial cells (p53, p21 and p16) was investigated. It was observed that the phosphorylation of ERK, but not of p38 and JNK, was correlated to the proliferation of endothelial cells induced by the knockdown of KNDC1. The increased expression of eNOS indicated that the activity of the endothelial cells increased; however, the expression levels of VCAM-1 and ICAM-1 did not change compared with NT-siRNA-transfected control cells. In addition, the phosphorylation of p53 and the expression of p21 and p16 decreased significantly compared with NT-siRNA-transfected control cells (Fig. 4).

\section{Discussion}

KNDC1 is a brain-specific Ras guanine nucleotide exchange factor. Overexpression of KNDC1 suppresses dendritic extension and branching in hippocampal neurons and cerebellar granule cells, whereas knockdown of endogenous KNDC1 expression promotes dendrite growth (11). However, the effect of KNDC1 on cellular senescence had yet to be elucidated. Cellular senescence, i.e., the limited ability of primary human cells to divide when cultured in vitro, is utilized as a model of biological aging. In common with other normal diploid cells, HUVECs have a limited capacity to divide (13). Therefore, in the present study, HUVECs were cultured in vitro as a model of biological aging, to investigate the effect of KNDC1 on senescence. It was observed that with increasing passages, the size of the senile HUVECs increased and the cells were positively stained with SA- $\beta$-gal. Furthermore, the transcription of the KNDC1 mRNA and expression of the KNDC1 protein increased with cellular senescence (Fig. 1). When the expression of KNDC1 was knocked down with a specific siRNA, the size and the number of the HUVECs decreased, and there was a statistically significant decrease in the number of HUVECs stained with SA- $\beta$-gal compared with control NT-siRNA-transfected HUVECs of the same passage. These results suggested that KNDC1 may be important in the progression of senescence in HUVECs and that knockdown of KNDC1 delayed this senescence. However, further investigation is required to determine whether overexpression of KNDC1 may accelerate senescence in HUVECs.

Cellular senescence is considered to be an irreversible block of cell cycle progression in populations of otherwise replication-competent cells $(14,15)$. The proportion of arrested cells in a population rises with increasing population doublings, rather than all cells becoming senescent at once $(16,17)$. In the present study, it was identified that knockdown of KNDC1 decreased the percentage of cells in the G0/G1 stage and increased the percentage of cells in the $\mathrm{S}$ and G2/M stages, compared with NT-siRNA-transfected control HUVECs from the same passage. The change in the percentage cells in $\mathrm{G} 0 / \mathrm{G} 1$ phase was similar to the change in the expression of KNDC1.

The alterations in morphology of HUVECs transfected with KNDC1-siRNA was minimal with regard to their function. Therefore, in order to obtain further insight into the effect of KNDC1 on angiogenesis, endothelial capillary tube network formation was investigated. The results demonstrated that when HUVECs were transfected with KNDC1-siRNA, the mean tubule length and the number of branch points was significantly increased compared with HUVECs transfected with NT-siRNA. These results indicated that knockdown of KNDC1 not only delayed senescence in HUVECs but also improved endothelial capillary tube network formation.

Following this, the mechanism by which transfection with KNDC1-siRNA altered HUVEC function was investigated. The MAPK signaling pathway is associated with cell proliferation $(18,19)$, eNOS, VCAM-1 and ICAM-1 are required for the function of $\operatorname{HUVECs}(20,21)$ and the p53-p21-p16 signaling pathway controls cell senescence $(22,23)$. In the present study, it was demonstrated that the knockdown of KNDC1 promoted HUVEC proliferation via the ERK signaling pathway, but not the p38 and JNK transduction cascades. Furthermore, knockdown of KNDC1 had no effect on the expression of VCAM-1 and ICAM-1, but increased the expression of eNOS, which induces the relaxation of blood vessels. In addition, the present study provided the first evidence, to the best of our knowledge, for the involvement of KNDC1 in the senescence of human primary endothelial cells, acting through the p53 signaling pathway. It was demonstrated that knockdown of KNDC1 delayed senescence in HUVECs by decreasing the phosphorylation of p53 and the expression of the associated downstream signaling molecules, p21 and p16.

In conclusion, knockdown of KNDC1 promoted the proliferation and delayed the senescence of HUVECs. Regarding the mechanism of action, it was demonstrated that knockdown of KNDC1 increased the percentage of cells in the $\mathrm{S}$ and $\mathrm{G} 2 / \mathrm{M}$ phases by inhibiting the p53-p21-p16 signaling pathway. In addition, it was observed that knockdown of KNDC1 increased the expression of eNOS and improved capillary tube network formation. Therefore, it is concluded that knockdown of KNDC1 contributed to delayed endothelial cell senescence. The results suggested that KNDC1 has the potential to be a novel target in the development of pharmacological agents to delay the aging process and extend human life.

\section{Acknowledgements}

This study was supported by a grant from the National Natural Science Foundation of China (no. 81001439).

\section{References}

1. Huang J, Gan Q, Han L, et al: SIRT1 overexpression antagonizes cellular senescence with activated ERK/S6k1 signaling in human diploid fibroblasts. PLoS One 3: e1710, 2008. 
2. Krouwer VJ,Hekking LH,Langelaar-Makkinje M,Regan-KlapiszE and Post JA: Endothelial cell senescence is associated with disrupted cell-cell junctions and increased monolayer permeability. Vasc Cell 4: 12, 2012.

3. Wang JC and Bennett M: Aging and atherosclerosis: mechanisms, functional consequences, and potential therapeutics for cellular senescence. Circ Res 111: 245-259, 2012.

4. Erusalimsky JD: Vascular endothelial senescence: from mechanisms to pathophysiology. J Appl Physiol (1985) 106: 326-332, 2009.

5. Cardus A, Uryga AK, Walters G and Erusalimsky JD: SIRT6 protects human endothelial cells from DNA damage, telomere dysfunction, and senescence. Cardiovasc Res 97: 571-579, 2013.

6. Huang J, Furuya A, Hayashi K and Furuichi T: Interaction between very-KIND Ras guanine exchange factor and microtubule-associated protein 2, and its role in dendrite growth - structure and function of the second kinase noncatalytic C-lobe domain. FEBS J 278: 1651-1661, 2011.

7. Pawson T and Nash P: Assembly of cell regulatory systems through protein interaction domains. Science 300: 445-452, 2003.

8. Ciccarelli FD, Bork P and Kerkhoff E: The KIND module: a putative signalling domain evolved from the $\mathrm{C}$ lobe of the protein kinase fold. Trends Biochem Sci 28: 349-352, 2003.

9. Mees A, Rock R, Ciccarelli FD, et al: Very-KIND is a novel nervous system specific guanine nucleotide exchange factor for Ras GTPases. Gene Expr Patterns 6: 79-85, 2005

10. Zeth K, Pechlivanis M, Samol A, Pleiser S, Vonrhein C and Kerkhoff E: Molecular basis of actin nucleation factor cooperativity: crystal structure of the Spir-1 kinase non-catalytic C-lobe domain (KIND)•formin-2 formin SPIR interaction motif (FSI) complex. J Biol Chem 286: 30732-30739, 2011.

11. Huang J, Furuya A and Furuichi T: Very-KIND, a KIND domain containing RasGEF, controls dendrite growth by linking Ras small GTPases and MAP2. J Cell Biol 179: 539-552, 2007.

12. Lin YJ, Zhen YZ, Wei J, Liu B, Yu ZY and Hu G: Effects of Rhein lysinate on $\mathrm{H} 2 \mathrm{O} 2$-induced cellular senescence of human umbilical vascular endothelial cells. Acta Pharmacol Sin 32: 1246-1252, 2011
13. Kim KS, Kim MS, Seu YB, Chung HY, Kim JH and Kim JR: Regulation of replicative senescence by insulin-like growth factor-binding protein 3 in human umbilical vein endothelial cells. Aging Cell 6: 535-545, 2007.

14. Jeyapalan JC and Sedivy JM: Cellular senescence and organismal aging. Mech Ageing Dev 129: 467-474, 2008.

15. Mao Z, Ke Z, Gorbunova V and Seluanov A: Replicatively senescent cells are arrested in G1 and G2 phases. Aging (Albany NY) 4: 431-435, 2012.

16. Passos JF, Saretzki G, Ahmed S, et al: Mitochondrial dysfunction accounts for the stochastic heterogeneity in telomere-dependent senescence. PLoS Biol 5: e110, 2007.

17. Tchkonia T, Morbeck DE, Von Zglinicki T, et al: Fat tissue, aging, and cellular senescence. Aging Cell 9: 667-684, 2010.

18. Fujikawa T, Shiraha H, Ueda N, et al: Des-gamma-carboxyl prothrombin-promoted vascular endothelial cell proliferation and migration. J Biol Chem 282: 8741-8748, 2007.

19. Cho H, Balaji S, Sheikh AQ, et al: Regulation of endothelial cell activation and angiogenesis by injectable peptide nanofibers. Acta Biomater 8: 154-164, 2012.

20. Miyauchi H, Minamino T, Tateno K, Kunieda T, Toko H and Komuro I: Akt negatively regulates the in vitro lifespan of human endothelial cells via a p53/p21-dependent pathway. EMBO J 23: 212-220, 2004

21. Kim KS, Kang KW, Seu YB, Baek SH and Kim JR: Interferon-gamma induces cellular senescence through p53-dependent DNA damage signaling in human endothelial cells. Mech Ageing Dev 130: 179-188, 2009.

22. Kida M, Sugiyama T, Yoshimoto $T$ and Ogawa $Y$ : Hydrogen sulfide increases nitric oxide production with calcium-dependent activation of endothelial nitric oxide synthase in endothelial cells. Eur J Pharm Sci 48: 211-215, 2013.

23. Murikipudi S, Methe H and Edelman ER: The effect of substrate modulus on the growth and function of matrix-embedded endothelial cells. Biomaterials 34: 677-684, 2013. 